\title{
Hey Joe, Jimi Hendrix Experience, 1966-1967
}

\author{
di Alberto Mario Banti
}

Lug 9, $2019 \mid \underline{\text { In evidenza, }}$ La colonna sonora di una generazione $\mid \underline{0 \mid}$

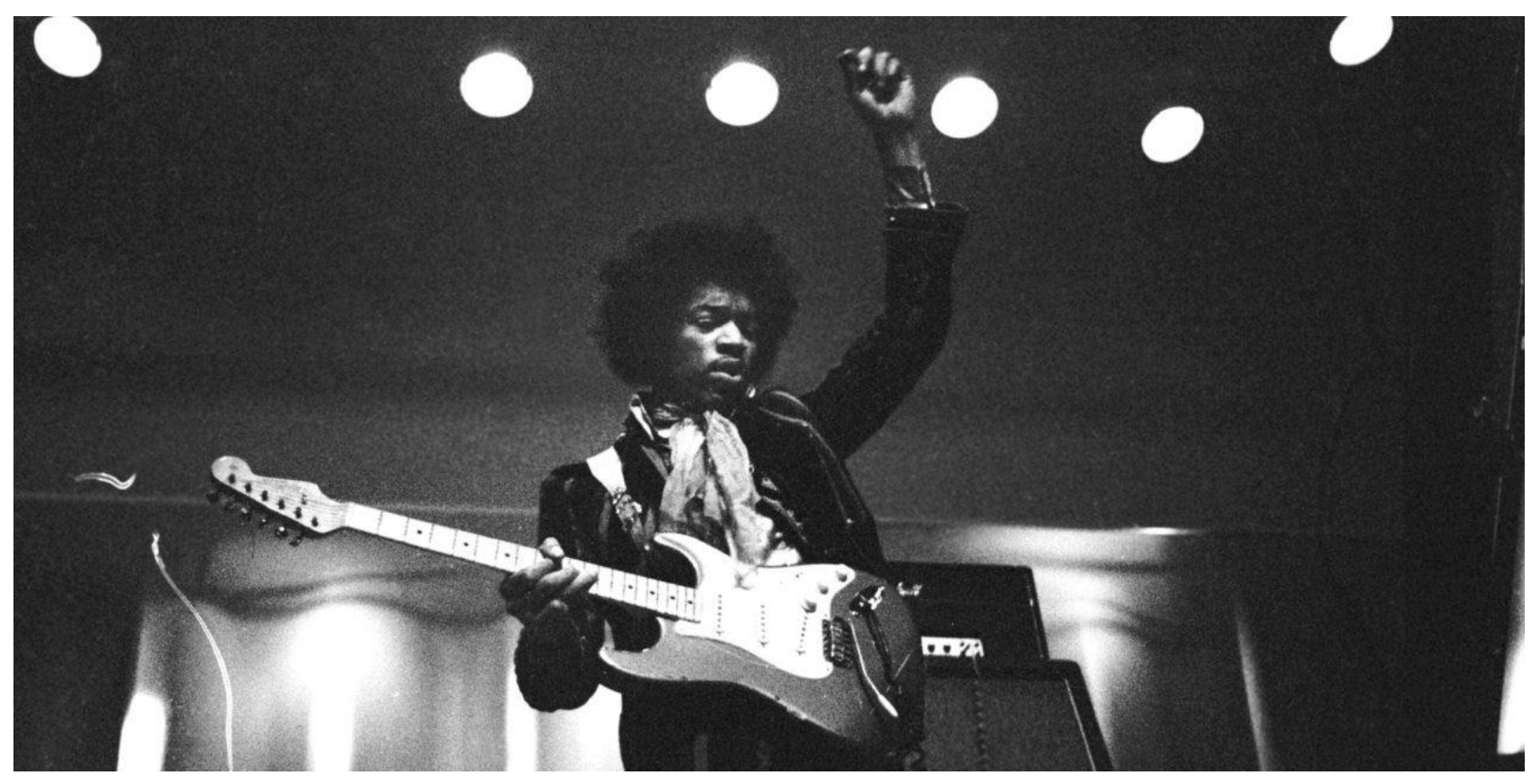

Jimi Hendrix a Helsinki nel 1967. Foto di Hannu Lindroos / Lehtikuva -

http://hs.mediadelivery.io/img/1920/9dbb99ea6c89473c9c8cec20806fa7b9.jpg, Public Domain,

Link

\begin{abstract}
Attraverso l'analisi e la contestualizzazione della canzone Hey Joe di Jimi Hendrix, l'autore ricostruisce genesi ed evoluzione del Rock nella metà degli anni Sessanta, soffermandosi particolarmente sul senso e sull'impatto del testo, sull'originario effetto shock delle narrative blues e sulla sensazione straniante di non-civiltà, evocata da un racconto come quello del classico hendrixiano, che non dovrebbe essere dimenticata e che dovrebbe aiutarci ad affrontare altre esperienze musicali care, negli anni seguenti, a varie e diverse audiences giovanili, dal punk, al grunge, allo hip-hop, alla trap che attualmente spopola tra gli adolescenti. Questo non per dire che ogni singola canzone che appartenga all'uno o all'altro universo sonoro abbia un valore in sé; ma per suggerire che, per specifiche audiences giovanili, esse possono avere un valore simbolico particolare, anche quando le storie raccontate sono perturbanti, anche quando i valori proposti sembrano volgari e repulsivi.
\end{abstract}

\section{In principio era il blues}

Che il rock - alle origini - sia profondamente influenzato dal blues è un dato piuttosto chiaro e assodato. Quali siano le implicazioni di questo fenomeno è una questione che - anche in una dimensione didattica - non deve sfuggire. 
Il blues classico, che nasce negli anni '30 del XX secolo nel Delta del Mississippi, è un universo poetico altamente inquietante; i racconti, espressi in soggettiva, cantati indifferentemente da voci maschili e femminili, esprimono un profondo senso di angoscia che è provocato da una qualche minaccia esterna: può essere qualcosa di molto concreto, tipo la povertà, l'alluvione, la siccità; ma può essere anche qualcosa di molto più indefinito (il «blues», il «jinx», il «devil») che perseguita il/la protagonista della canzone in ogni momento della sua giornata. Per sfuggire si può provare a viaggiare, salendo su un treno, e andando lontano; oppure si può provare a fare un «viaggio» di tipo diverso, con l'alcol o con la droga. In nessuno dei due casi ci si sottrae davvero alla minaccia: il treno non porta da nessuna parte; l'alcol e la droga conducono all'autodistruzione [1].

\section{TUTTO HA UNA BRUTTA FINE}

Niente va bene nelle storie blues, anche se di tanto in tanto questa angoscia viene convertita in humor nero: non vanno bene gli amori, destinati a finir male, e non di rado in forme violente; non va bene l'esperienza esistenziale nel suo complesso, che talora è talmente deragliata da spingere i protagonisti dei racconti a commettere qualche crimine, finire in carcere, essere condannati a morte. Uno dei pochi sollievi a questa inquietudine incessante è la sessualità, vissuta spesso in modo molto diretto e molto carnale.

Ora è chiaro che un universo poetico di questo tipo è immediatamente collegato alle terribili condizioni di vita delle comunità afroamericane del sud-est degli Stati Uniti, là dove il blues originariamente nasce. Ma c'è un aspetto che rende piuttosto universale questa musica, altrimenti così legata a esperienze molto precise: il disagio esistenziale viene espresso spesso in forme indeterminate, prive di coordinate geografiche o sociali precise. Specie quando si canta del «blues», cioè dell'astratta angoscia che opprime un individuo, si ricorre a figure tanto suggestive quanto indefinite, come fa, per esempio, Robert Johnson in Hellhound on My Trail (1937):

Mi devo muovere, mi devo muovere, i blues vengon giù come grandine, i blues vengon giù come grandine

Umm mmm mmm, i blues vengon giù come grandine, i blues vengon giù come grandine

E il giorno continua a tormentarmi, ho un segugio infernale alle calcagna, un segugio alle calcagna, un segugio alle calcagna [2]

L'immagine del «segugio infernale» ha lo stesso valore del «blues», del «jinx», del «devil»: figure dell'angoscia, che hanno un carattere indeterminato, e al tempo stesso orribilmente concreto, nella descrizione dell'infinita inquietudine che percorre la musica blues.

\section{Superare la "linea del colore"}

Nel contesto degli USA degli anni '30-'50 la musica blues non esce dallo spazio delle comunità afroamericane: neri i musicisti o le musiciste; nero il pubblico di riferimento. Non serve a infrangere questa dura «linea del colore» la nascita del blues elettrico, che prospera a Chicago negli anni '50, con l'emergere di musicisti come Muddy Waters o B.B. King; e non aiuta nemmeno l'influenza che il blues esercita su un nuovo genere di gran moda in quello stesso periodo, il r'n'r, che seppure utilizza le forme musicali del blues, ne ignora tuttavia completamente l'universo poetico [3].

Affinché il blues possa superare la «linea del colore», c'è bisogno di spostarsi in un altro posto e in un altro tempo: il Regno Unito dei primi anni '60. Lì arrivano facilmente molte musiche che vengono dagli USA, sia perché la barriera linguistica non esiste, sia perché le basi militari americane presenti sul paese diffondono facilmente la conoscenza della produzione discografica 
americana, attraverso le trasmissioni radio o la circolazione dei vinili. In questo contesto alcuni giovani musicisti britannici trovano nel blues una forma musicale, uno stile vocale e un mondo narrativo assolutamente mai sentito prima nella pop music degli anni precedenti e per questo profondamente emozionante; vi trovano infatti qualcosa che permette loro di esprimere, in forme nuove e profonde, il loro disagio di ragazzi spesso proveniente da ambienti sociali e familiari piuttosto disagiati. Secondo John Steel, il batterista degli Animals, il duro panorama industriale di Newcastle, città dalla quale il gruppo proviene, lo hanno spinto verso «un'istintiva identificazione emotiva con il blues nero americano». Dal canto suo Eric Clapton, figlio illegittimo allevato dai nonni, ha dichiarato:

[da ragazzo] mi sentivo con le spalle al muro e pensavo che l'unico modo di sopravvivere era con dignità, orgoglio e coraggio. Ho sentito tutto ciò in certe forme di musica, e in particolare l'ho sentito nel blues... Si trattava di un uomo e la sua chitarra contro il mondo... quando si arrivava al dunque, si trattava di un tizio completamente solo e senza possibilità, senza alternative, se non di cantare e suonare per placare i propri dolori. E tutto ciò rispecchiava quello che sentivo [4].

\section{IL BLUES, OVVERO LA “ROBA CHE SUCCEDE ALLA GENTE"}

Altri apprezzano la «autenticità» del blues: Bill Wyman, a lungo bassista dei Rolling Stones, ha detto: «Tutte quelle canzoni melense sul rossetto che ha macchiato il tuo colletto e roba così, erano veramente risibili... ma quando qualcuno cantava del suo lavoro sulla ferrovia a un dollaro al giorno... lo potevi apprezzare, perché probabilmente l'aveva fatto davvero... era ciò che accadeva nella vita». Ed Eric Burdon, leader degli Animals, ha affermato che la prima volta che ha sentito Five Long Years (un blues del 1952, scritto da Eddie Boyd) ha pensato: «Questa è roba che succede alla gente... agli uomini cresciuti nel mio quartiere» [5]. D'altronde il blues ha un notevole appeal anche per le ascoltatrici britanniche, che quando scoprono le canzoni di Bessie Smith o di Gertrude «Ma» Rainey (due grandi cantanti blues afroamericane) riescono a trovarci un'infinità di suggestioni, sia che le blueswomen cantino il loro disagio affettivo, sia che cantino di una qualche loro brusca reazione all'insensibilità o alla superficialità dell'uomo amato [6].

\section{USA-UK, ANDATA E RITORNO}

In qualche caso vi sono dei giovani musicisti britannici, come John Mayall o Alexis Korner o Cyril Davies, che fanno cover di classici blues o scrivono musiche e testi originali rispettando religiosamente le canoniche regole musicali e poetiche del blues. Altri musicisti, come gli Yardbirds, gli Animals o i Rolling Stones, pur facendo cover di blues, scrivono anche musiche e testi originali ispirati al blues, ma contaminati con altre forme musicali (il R\&B, il country, il folk, talora persino il jazz), cosa che consente loro di ampliare i loro orizzonti musicali.

La novità e l'esoticità dei loro brani attraggono l'attenzione di giovani ascoltatori e ascoltatrici in UK. Poi, sulla scia del successo dei Beatles, che dal 1964 in avanti si impongono anche nelle classifiche USA, riescono ad avere successo anche negli Stati Uniti, in tal modo facendo accettare al pubblico bianco americano delle forme musicali e narrative che prima erano considerate del tutto inaccettabili perché provenienti dalle comunità afroamericane. In tutto ciò si può forse vedere un esempio dell'atteggiamento predatorio che la società bianca ha nei confronti della cultura afroamericana; ma vi si può anche vedere un serio e sincero omaggio a quella cultura, che come effetto collaterale ha anche quello di rilanciare presso il pubblico bianco degli USA la carriera di musicisti blues afroamericani - come Muddy Waters, B.B. King, Howlin' Wolf, e altri ancora - fin allora confinati nel «ghetto» della cosiddetta «race music». 


\section{Hey Joe}

Dunque, è attraverso questo percorso che si pongono le fondamenta della nuova musica rock, influenzata dalle forme musicali blues e soprattutto dalle forme narrative di questo genere musicale. È ciò che si può vedere molto chiaramente in uno dei successi del rock delle origini, ovvero Hey Joe, brano contenuto nel primo 45 giri della Jimi Hendrix Experience, pubblicato in UK nel dicembre del 1966, e poco più tardi - nel 1967 - anche negli USA [7]. Il brano ha una struttura e una storia complessa; viene scritto nel 1962 da un busker bianco che si esibisce a New York e in California, Billy Roberts, che non lo incide mai; originariamente ha un impianto country, ed esercita un fascino talmente forte che tra il 1965 e il dicembre del 1966, quando esce la versione di Jimi Hendrix, la canzone è già stata incisa altre sette volte da altrettanti gruppi o musicisti che ne offrono versioni in stile beat o folk.

Hendrix, invece, trasforma il brano - sia nella struttura ritmica, sia nella versificazione - in un vero e proprio blues, operazione che è resa possibile anche dalla storia che viene raccontata nel testo, che potrebbe senz'altro derivare - almeno come spirito - dall'universo testuale del blues. Ed è una storia massimamente inquietante:

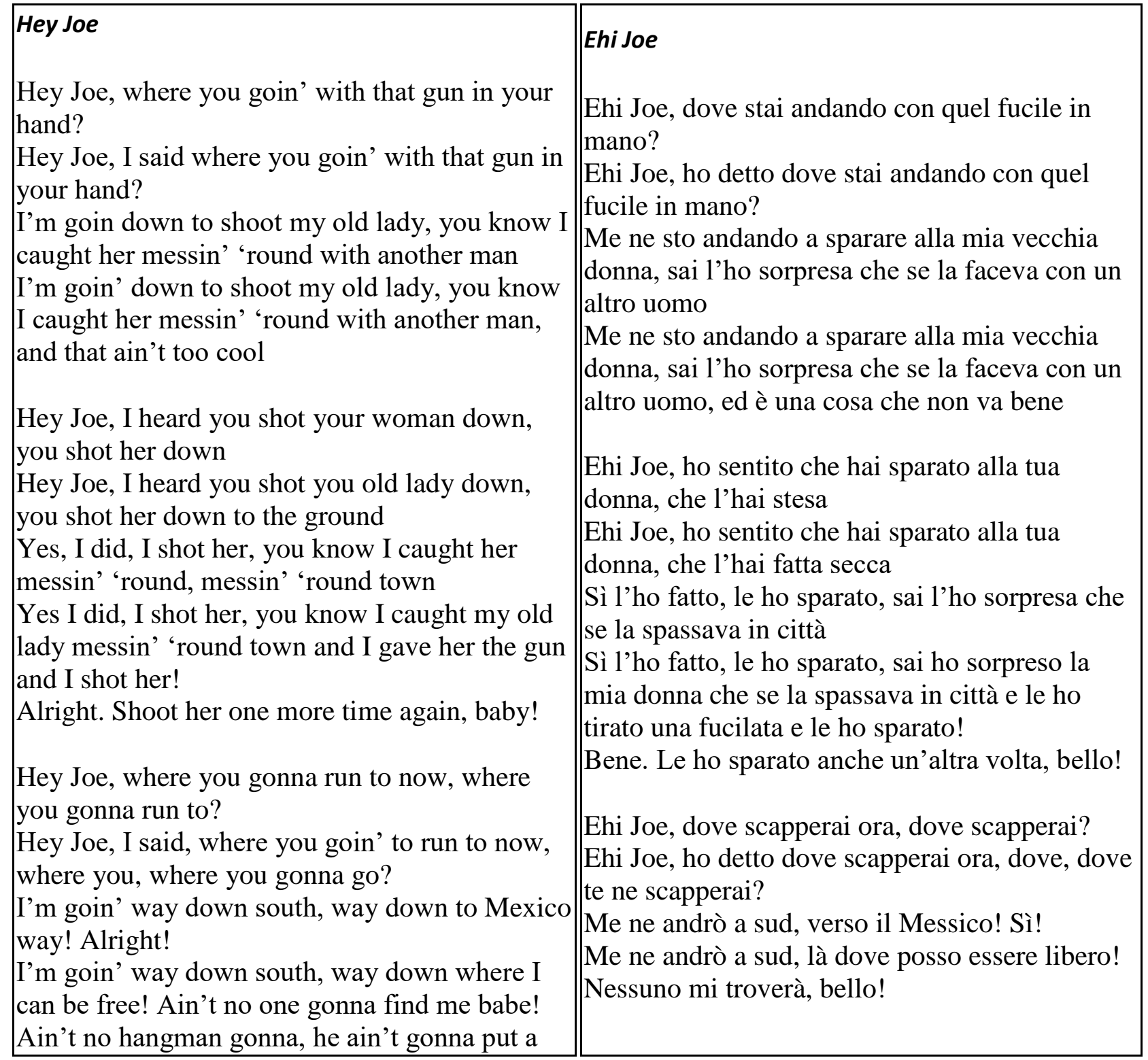


rope around me!

You better believe it right now! I gotta go now!

Hey, hey, hey Joe, you better run on down!

Goodbye everybody. Ow! Hey, hey Joe ...
Nessun boia mi, lui non mi metterà un nodo al

collo!

È meglio che tu ci creda proprio ora! Me ne andrò ora!

Ehi, ehi, ehi Joe, meglio che te ne scappi!

Addio a tutti. Ehi, ehi Joe ...

\section{UNA STORIA “FUORI DAL TEMPO"}

Nel 1967 Hey Joe, nella versione di Jimi Hendrix, si impone nell'immaginario giovanile statunitense ed europeo. Quando comincia a circolare, l'atmosfera sociale e politica negli USA e in Europa è ormai molto tesa. I soldati americani che dal 1964 combattono in Vietnam sono ormai quasi 500.000. Le manifestazioni pacifiste, negli USA e in Europa, investono le università e le strade delle grandi città. Il movimento per i diritti civili degli afroamericani vive un momento di crisi, con la nascita di gruppi che vorrebbero rinnegare la linea non violenta seguita negli anni precedenti da Martin Luther King. Intanto le tensioni nei ghetti neri delle grandi città americane esplodono più volte in risposta a interventi brutali della polizia: gravi rivolte scoppiano il 18 luglio 1964 a Harlem; tra l'11 e il 17 agosto del 1965 a Watts, un quartiere di Los Angeles; nel 1967 a Detroit, Newark, Tampa, Cincinnati, Milwaukee, Atlanta [8].

Ebbene, di tutto ciò, nel brano-simbolo della Jimi Hendrix Experience non vi è traccia. La storia sembra appartenere a un altro tempo e a un altro mondo. Raccontata in forma di dialogo, narra di un uomo che passa con un fucile in mano mentre qualcuno che lo conosce gli chiede: «Ehi Joe, dove vai?»; e lui: «Me ne vado a far secca la mia donna; sai, se la fa con un altro». Quando Joe torna indietro, l'amico gli chiede: «Ehi, ma è vero che hai steso la tua donna?»; e Joe risponde: «Certo che le ho sparato, l'ho sorpresa in città che se la stava spassando. E non solo le ho sparato una volta, ma due volte!». «E ora dove te ne vai?» - replica l'altro. «Scappo in Messico; stai pur sicuro, lì nessuno mi metterà un cappio al collo»; «Beh, Joe, allora è meglio che ti muova», commenta l'osservatore.

Strana storia per i tempi che corrono: non ci sono eroi luminosi; non ci sono valori positivi; non c'è alcun riferimento al contesto contemporaneo; c'è «solo» un «femminicidio», come diremmo oggi, descritto nel più indifferente dei modi. Quindi, che significato possiamo attribuire a una canzone di questo tipo? In forma generica si può anche osservare che il testo trasuda inquietudine, e persino rabbia a stento trattenuta. Ma perché inquietudine e rabbia dovrebbero essere espresse proprio in questo modo? E perché la canzone è apprezzata da un così vasto pubblico giovanile, in Europa e poi anche negli Usa?

\section{Il Rock: un'elettrizzante libertà}

La risposta va cercata nelle ragioni per le quali giovani musicisti come Clapton, Harrison, Jagger, Richards o Mayall hanno cominciato ad apprezzare il blues. Ispirandosi a quella musica si possono raccontare storie che la cultura di massa mainstream (cinema hollywoodiano, format televisivi, pop songs di successo) non racconta: storie inquiete, che smentiscono l'ottimismo di facciata che la cultura di massa dominante vorrebbe imporre attraverso vicende aggraziate e di buoni sentimenti come quelle raccontate per esempio in Mary Poppins o in Tutti insieme appassionatamente (due successi cinematografici rispettivamente del 1964 e del 1965). Si possono raccontare storie che reintroducono il tragico nella cultura di massa. E lo si fa senza assumere l'atteggiamento moralistico e manicheo che è proprio della cultura di massa dominante (il bene è il bene, il male è il 
male, collocati in due campi nettamente distinti, e il bene - attraverso i personaggi che lo incarnano - alla fine invariabilmente trionfa, perché deve trionfare).

In canzoni come Hey Joe non c'è alcun atteggiamento pedagogico o morale. La storia non vuole spiegare a chi ascolta dove sta il bene e dove sta il male. Il testo racconta una storia possibile: poi sta a chi ascolta posizionarsi eticamente, se vuole faro; trarne le sue personali considerazioni; riflettere su quello che, in effetti, può succedere intorno, in qualche periferia urbana, come anche in qualche attico upper-class. Per ragazzi e ragazze abituate a produzioni che non lasciano spazi a dubbi, trovarsi davanti a canzoni come Hey Joe dev'essere stato come provare un'elettrizzante sensazione di libertà: liberi di ascoltare storie strane; liberi di avere una visione più contrastata del mondo; liberi di scegliere autonomamente in cosa credere, quale posizione prendere, che reazione avere.

Con tutto ciò, ci sono almeno due aspetti ancora che vanno sottolineati.

\section{DOV'È LA POLITICA?}

Il primo: molte delle canzoni rock non hanno un contenuto esplicitamente politico, non sostengono direttamente qualche partito, o movimento, o leader; e nonostante ciò conservano un contenuto controculturale che in gran parte deriva dal dialogo con un archivio narrativo ricco e eterodosso come quello del blues; dopodiché, il rock è una musica aperta a ibridazioni di ogni tipo che arricchiscono questo nucleo narrativo originario; tuttavia è il nucleo narrativo blues che conferisce al rock delle origini il suo valore alternativo e trasgressivo [9].

\section{ROCK MASCHILISTA?}

Secondo aspetto: leggendo Hey Joe si potrebbe essere inclini ad accogliere l'opinione di chi ritiene che il rock sia un genere musicale maschilista - sia per la prevalenza di musicisti maschi, sia per come vengono affrontati i rapporti di genere. Ora, posto che il tema meriterebbe una riflessione a parte, qui c'è almeno da osservare che una valutazione critica di questo tipo, applicata a Hey Joe, porta a conclusioni sbagliate. Nell'archivio narrativo blues ci sono tante storie di amori che finiscono male, in modo violento, anche con un omicidio, e sono cantate indifferentemente da cantanti maschi e da cantanti femmine (come Bessie Smith o Victoria Spivey, per es.). Lo stesso succede nel rock che dialoga col blues. Proprio nello stesso anno in cui esce Hey Joe viene lanciato anche il primo LP dei Jefferson Airplane, importante rock band californiana (Jefferson Airplane Takes Off, agosto 1966): l'album contiene una cover di un brano blues di Memphis Minnie, originariamente registrato nel 1941, Chauffeur Blues; i Jefferson Airplane lo affidano alla voce, bella e spavalda, della loro cantante, Signe Toly Anderson, che canta di una lei che vuole che lui sia il suo autista, e che la porti in giro per il mondo: ma se il suo lui provasse a portare in giro delle altre ragazze, beh allora lei è pronta a sparargli [10]. Dal canto suo Janis Joplin, una musicista rock che dialoga intensamente con la tradizione blues, esplora spesso il registro autocommiserante che è proprio di quel tipo di musica, raccontando storie in cui una donna vittimizzata dal suo uomo vive storie d'amore tristi e disperate; ma è anche capace di capovolgere questo schema, per esempio in brani come Turtle Blues (1968), in cui dice: «non sono il tipo di donna che trasforma la vita degli uomini in un letto di rose: vuoi dire per questo che sono cattiva? beh, fa' un po' come ti pare, mi han già chiamato in così tanti modi; quello che è certo è che mi prenderò buona cura di me stessa, e nessuno mi metterà mai sotto». E in esecuzioni dal vivo di classici blues, la virata narrativa è ancora più netta: in Black Mountain Blues (brano originariamente cantato da Bessie Smith), lei, abbandonata da lui, non ci mette niente a prendere un fucile e un coltello e ad annunciare (a se stessa o alla sua comunità): «Gli sparerò se starà fermo, lo accoltellerò se proverà a scappare»; e in Careless Love (altro classico blues) il tradimento costa la vita a lui e alla sua amante: «Maledetto, 
ora io ti sparerò, Dio, vi ammazzerò entrambi ora / Non risparmierò nessuno di voi fino a quando sarete davvero morti» [11].

\section{Conclusioni}

In conclusione: adesso ascoltiamo Hey Joe col rispetto che si tributa a un classico, e non ci si interroga poi molto sul senso e sull'impatto del testo. Eppure l'originario effetto shock delle narrative blues non dev'essere dimenticato. La sensazione straniante di non-civiltà, evocata da un racconto come quello del classico hendrixiano, non dovrebbe essere dimenticata e dovrebbe aiutarci ad affrontare altre esperienze musicali care, negli anni seguenti, a varie e diverse audiences giovanili, dal punk, al grunge, allo hip-hop, alla trap che attualmente spopola tra gli adolescenti: questo non per dire che ogni singola canzone che appartenga all'uno o all'altro universo sonoro abbia un valore in sé; ma per suggerire che, per specifiche audiences giovanili, esse possono avere un valore simbolico particolare, anche quando le storie raccontate sono perturbanti, anche quando i valori proposti sembrano volgari e repulsivi. Prima di abbandonarci a un giudizio morale, abbiamo sempre il dovere di comprendere: e l'esempio di Hey Joe può aiutarci ad apprezzare il valore di una ricostruzione analitica rispetto alla scorciatoia della ripulsa morale, per quanto giustificata essa possa sembrare al primo sguardo.

Note:

[1] Adam Gussow, Seems Like Murder Here. Southern Violence and the Blues Tradition, University of Chicago Press, Chicago and London 2002; R.A. Lawson, Jim Crow's Counterculture. The Blues and Black Southerners, 1890-1945, Louisiana State University Press, Baton Rouge 2010; Vincenzo Martorella, Il Blues, Einaudi, Torino 2009; Allan Moore(ed.), The Cambridge Companion to Blues and Gospel Music, Cambridge University Press, Cambridge 2002.

[2] Luigi Monge, Robert Johnson. I Got The Blues. Testi commentati, Arcana, Roma 2008, p. 191.

[3] Alberto Mario Banti, Wonderland. La cultura di massa da Walt Disney ai Pink Floyd, Laterza, Roma-Bari 2017, capp. VI-VII.

[4] Roberta Freund Schwartz, How Britain Got the Blues. The Transmission and Reception of American Blues Style in the United Kingdom, Ashgate, Aldershot-Burlington 2007, p. 74.

[5] Cit. ivi, p. 136. Lipstick on Your Collar è una canzone cantata da Connie Francis, che nel 1959 va in cima alla classifica delle vendite nel Regno Unito, negli Usa e in Australia.

[6] Ivi, pp. 74-75.

[7] Charles R. Cross, La stanza degli specchi. Jimi Hendrix: la vita, i sogni, gli incubi, Feltrinelli, Milano 2010 [2005], p. 217.

[8] Bruno Cartosio, I lunghi anni sessanta. Movimenti sociali e cultura politica negli Stati Uniti, Feltrinelli, Milano 2012, pp. 117-125.

[9] Banti, Wonderland, cit., pp. 423-434. 
[10] Sin dal primo album i brani dei Jefferson Airplane sono cantati sia dalla cantante (prima Signe Toly Anderson; poi la carismatica Grace Slick), sia da uno dei chitarristi (Marty Balin, Paul Kantner, Jorma Kaukonen). Memphis Minnie (18971973) è una brillante musicista blues, con una carriera ricca e longeva. Per una panoramica della carriera dei Jefferson si veda Jeff Tamarkin, Got a Revolution! The Turbulent Flight of Jefferson Airplane, Atria Books, New York 2003.

[11] Massimo Cotto (a cura di), Cry Baby. I testi di Janis Joplin, Arcana, Roma 2007, pp. 169 e 177; cfr. anche Alice Echols, Graffi in Paradiso. La vita e i tempi di Janis Joplin, Arcana, Roma 2010 [1999]. 